\title{
EDITORIAL
}

\section{QUANDO A FIBROSE HEPÁTICA SE TORNA DOENÇA...}

Inúmeros exemplos em patologia ilustram ofato de que toda perda celular gera uma resposta de substituição por tecido conjuntivo. Trata-se de uma resposta de adaptação transitória ou permanente, de caráter cicatricial. Em outros casos, pelo contrário, existe uma resposta completamente diferente: há uma ativação do tecido conjuntivo que leva ao que se chama comumente uma doença fibropoliferativa ${ }^{2}$. Nesta circunstância trata-se de uma resposta adaptativa "inadaptada" ou de uma doença do tecido conjuntivo?

Em patologia hepática, qualquer que seja o tipo de resposta, é realmente a fibrose que gera complicações graves: a fibrose é efetivamente a responsável direta pelo agravamento da insuficiência hepatocelular e da hipertensão porta.

Os progressos mais significativos nos últimos anos no estudo da fibrose resultam do conhecimento cada vez mais preciso do que se chamava outrora "a substância fundamental" (terminologia espantosamente preditiva). Esta é composta de mais de 25 micromoléculas, agora isoladas, purificadas, seqüenciadas em numerosas espécies animais e humanas. Podem ser citadas quantitativamente como principais isotipos de colágeno ${ }^{8}$ : os proteoglicanos, a fibronectina, a elastina, a laminina e a entactina.

A noção durante muito tempo restritiva da existência de uma só população de células conjuntivas, originando-se da secreção dos componentes das matrizes, já é quase histórica. $O$ dogma da exclusividade deste controle cedeu pela evidência de produtos de síntese precursores das proteinas das matrizes e, mais recentemente, pelas demonstrações que sustentam a expressão gênica destas células. Estas evidências foram obtidas por imunomarcação intracelular em tipos celulares variados de células epiteliais - parenquimatosas ${ }^{1}$

$\mathrm{Na}$ fibrose hepática, os arranjos estruturais observados resultam de uma cascata de acontecimentos em cadeia, levando à perturbação dos equilíbrios $\mathrm{e}$ das interações células-matriz conjuntiva.

Várias unidades de pesquisa têm demonstrado nos últimos anos, com diferentes modelos, que a matriz conjuntiva não é somente uma estrutura de sustentação; ela nos revela um papel integrador fundamental. Em patologia, sabemos agora que seu polimorfismo estrutural lhe deu notavel plasticidade, gerando tanto "perfis protéicos" para diferentes matrizes, como estados evolutivos lesionais: tudo leva a

Recebido para publicação em 6/11/87.

\section{QUAND LA FIBROSE HÉPATIQUE DEVIENT UNE MALADIE...}

On ne compete plus les exemples en pathologie illustrant le fait que toute perte cellulaire est génératrice d'une réponse de comblement par le tissu conjonctif. Il s'agit d'une réponse adaptative transitoire ou permanente, à caractère cicatriciel. Dans d'autres cas en revanche, s'exprime une réponse toute différente: on assiste à une activation du tissu conjonctif qui aboutit à ce que l'on appelle communément une maladie fibroproliférative $e^{2}$. S'agit-il alors d'une réponse adaptative "inadaptée" ou d'une maladie du tissu conjonctif? En pathologie hépatique, et quel que soit le type de réponse, c'est bien la fibrose qui est génératrice des complications graves: la fibrose est en effet directement responsable de l'aggravation de l'insuffisance hépatocellulaire et de l'hypertension portale.

Les progrès les plus significatifs de ces dernières années dans l'étude de la fibrose sont le résultat de la connaissance de plus en plus précise de ce que l'on appelait autrefois "la substance fondamentale" (terminologie étonnamment prédictive) composée de plus de 25 macromolécules maintenant isolées, purifiées, séquencées dans de nombreuses espèces animales et chez l'Homme parmi lesquelles les isotypes de collagène $^{8}$, les protéoglycannes, la fibronectine, l'élastine, la laminine et l'entactine sont quantitativement les principales.

La notion longtemps restrictive de l'existence d'une seule population de cellules conjonctives à l'origine de la sécrétion des composants matriciels est devenue presque historique. Le dogme de l'exclusivité de ce contrôle a cédé devant la mise en évidence, par immunomarquage intracellulaire ${ }^{1}$ au niveau de types cellulaires variés dont les cellules épithéliales - parenchymateuses -, de produits de synthèse précurseurs des protéines matricielles et, plus récemment, de démonstrations portant sur l'expression génique de ces cellules.

Dans la fibrose hépatique, les remaniements structuraux observés sont le résultat d'une cascade d'évèments en chaîne aboutissant au bouleversement des équilibres et des interactions cellules-matrice conjonctive.

Plusieurs unités de recherche ont démontré ces dernières années que, sur différents modèles, la matrice conjonctive n'est pas seulement une structure de soutien; elle nous dévoile un rôle intégrateur fondamental. En pathologie, nous savons maintenant que son polymorphisme structural se double d'une plasticité très remarquable offrant autant de "profils protéiques" matriciels différents que de stades évolutifs lésionnels: tout porte à supposer qu'à travers son ré- 
supor que através de sua rede transitam, param, se modificam ou desaparecem sinais (mediadores) e mensagens entre as populações celulares ${ }^{3}$. Há um verdadeiro papel modulador da matriz do conjuntivo nas trocas e interaçōes celulares. Assim, os constituintes da matriz são ao mesmo tempo os suportes e os autores do arranjo tissular na fibrose.

Uma análise mais fina da fibrose e de seu entendimento implica, obrigatoriamente, o desenvolvimento de método de exploração mais sofisticado do que dispomos no presente.

Ao nível tissular, depois de muitos anos, a obtenção de anticorpos específicos permitiu a localização pela imunofluorescência de diferentes isotipos de colágeno ${ }^{7}$ e proteínas associadas. Ainda persistem muitos pontos obscuros sobre o papel de cada um dos constituintes tissulares na fibrose. Contudo, a imunofluorescência já permite uma abordagem mais dinâmica: trata-se de uma fibrose ainda jovem com predominância de colágeno de tipo III ou de uma fibrose bem instalada constituida de fibras grossas de colágeno de tipo I? Há uma evolução para a capilarização dos sinuisóides com depósito de proteinas especificas das membranas basais...?

Também, ao nivel tissular, os métodos de dosagem quantitativa do colágeno têm sido miniaturizados e a ausência de extração prévia os torna mais confiáveis: assim, é possivel medir de modo simples a quantidade de colágeno total de um corte histológico de fígado, graças à afinidade do vermelho Sirius para o colágeno $^{12}$, ou de medir a quantidade dos diferentes isotipos, graças a anticorpos especificos marcados pela peroxidase 9 . Ao invés de se calcular subjetivamente a fibrose em um corte histológico pode-se fazer a medida ponderal dos diferentes tipos de colágeno e estabelecer, facilmente, uma proporção dos diferentes tipos tissulares de colágeno.

No entanto, a biópsia hepática não pode ser proposta como método de avaliação em populações com al to risco de fibrose, em razão dos possiveis acidentes. E nem pode ser repetida freqüentemente nos doentes em evolução. Ademais, a ausência de homogeneidade da fibrose tissular pode ser responsável por uma certa variabilidade na composição entre uma e outra amostra. Por todas estas razōes, há muitos anos tem-se procurado ativamente marcadores séricos de fibrose hepática ${ }^{10}$. Estas pesquisas têm sido orientadas em duas direções principais: de uma parte, a avaliação indireta pela medida da atividade sérica de diferentes enzimas implicadas na síntese ou na degradação do colágeno; e de outra parte, avaliação mais direta pela medida das taxas circulantes de diferentes isotipos de colágeno ou de produtos de degradação ${ }^{11}$.

Atualmente, o único método fácil e o mais utilizado é a dosagem radioimunológica do P-III-P (propeptideo amino-terminal do procolágeno de tipo III) ${ }^{2}$. seau et qu'au cours du temps, signaux (médiateurs) et messages entre les populations cellulaires transitent $s$ 'arrêtent, se modifient ou disparaissen $t^{3}$. Un véritable rôle modulateur de la matrice conjonctive dans les échanges et interactions cellulaires apparait. Ainsi, les constituants matriciels sont à la fois les support et les acteurs du remaniement tissulaire dans la fibrose.

Une analyse plus fine de la fíbrose et un dépistage plus précoce passent obligatoirement par le développement de méthodes d'exploration plus élaborées que celles dont nous disposions jusqu'à présent.

$\mathrm{Au}$ niveau tissulaire, l'obtention d'anticorps spécifiques a permis depuis quelques annees la localisation par immunofluorescence des différents isotypes de collagène ${ }^{7}$ et protéines associées. Beaucoup d'inconnues persistent encore sur le rôle de chacun des constituants tissulaires dans la fibrose, mais l'immunofluorescence permet d'ores et déjà une approche plus dynamique: s'agit-il d'une fibrose encore jeune avec prédominance de collagène de type III ou d'une fibrose bien installée constituée de grosses fibres de collagène de type I?, y a-t-il une évolution vers la capillarisation des sinusoïdes avec dépôt de protéines spécifiques des membranes basales...?

Toujours au niveau tissulaire, les méthodes de dosage quantitatif du collagène ont été miniaturisées et l'absence d'extraction préalable les rend plus fiables: il est ainsi possible de mesurer très simplement la quantité de collagène total d'une coupe histologique de foie grâce à l'affinité du rouge Sirius pour le collagène ${ }^{12}$ ou de mesurer la quantité des différents isotypes sur coupe flottante grâce à des anticorps spécifiques marqués à la péroxydase ${ }^{9}$. A la notion de score histologique de fibrose va donc se substituer bientôt une mesure ponderále des différents types de collagène, et un rapport des différents types tissulaires de collagène pourra alors être facilement établi.

Cependant, la biopsie hépatique ne peut pas être proposée, en raison d'accidents toujours possibles, comme méthode de surveillance des populations à haut-risque de fibrose, ou ne peut pas être répétée fréquemment chez les malades déjà expertisés et dont on veut suivre l'évolution. En outre, l'absence d'homogénéité de la fibrose tissulaire peut être responsable d'une certaine variabilité dans la composition d'un échantillon à l'autre. Pour toutes ces raisons, des marqueurs sériques de fibrose hépatique ${ }^{10}$ ont été recherchés activement depuis plusieurs années. Ces recherches ont été menées dans deux directions principales: d'une part, appréciation indirecte par la mesure de l'activité sérique de différentes enzymes impliquées dans la synthèse ou la dégradation du collagène; d'autre part, appréciation plus directe par la mesure des taux circulants des différents isotypes de collagène ou de produits de dégradation ${ }^{11}$. 
Editorial. Grimaud JA, Druguet $M$, Hartmann D. Quand la fibrose hépatique devient une maladie.. Revista da Sociedade Brasileira de Medicina Tropical 20: 189-192, Out-Dez, 1987

Com efeito, as dosagens de atividade enzimática são ainda tecnicamente delicadas e muitas vezes sujeitas a erros. Por outro lado, a atividade enzimática circulante é rapidamente destruida ou inibida. Resultados interessantes, pelo contrário, têm sido descritos com a dosagem do P-III-P: a superposição de valores individuais obtidos em diferentes patologias hepáticas dificulta a utilização deste teste com fins diagnósticos. Entretanto, associado a outros marcadores, pode ser uma ajuda ao diagnóstico que permite, por exemplo, diferenciar a simples esteatose da hepatite alcoólica aguda no curso da intoxicação alcoólica, ou ainda, em caso de hepatite viral crônica, de seguir a evolução para a hepatite crônica ativa ${ }^{5} 14$. Com efeito, em geral, a taxa sérica de $P$-III-P parece ser mais um marcador da atividade da fibrose do que de sua quantidade. Isto explica as discordâncias verificadas nos estudos sobre cirrose hepática: com efeito, a taxa será elevada em caso de aposição recente de colágeno de tipo III e portanto de fibrose ativa, mas não será elevada em caso de fibrose instalada onde predomina o colágeno de tipo $I$. Outros peptideos circulantes ainda dependem de confirmação e poderiam completar, em breve, a gama dos testes disponiveis: procolágeno I, fragmentos de colágeno de tipo IV (7S, NC1).

Na prática, até o presente, nenhum estudo clínico foi feito em um grupo homogêneo de pacientes, seguido durante certo tempo, e avaliado simultaneamente com todos os marcadores de fibrose disponiveis, tissulares e séricos. É necessário desenvolver este tipo de estudo, mas desde já se pode afirmar que o clínico não mais pode adotar uma atitude resignada diante da fibrose hepática. A fibrose hepática è uma doença autonoma, que deve ser descoberta e avaliada e que em futuro próximo poderá ser tratada: com efeito, resultados interessantes têm sido descritos, pelo menos em animal, com novas moléculas potencialmente antifibrosantes.

Em conclusão, parece claro que as pesquisas agora convergem para três objetivos:

- Estabelecer os mecanismos celulares e moleculares que regulam, in vivo, a natureza do depósito na matriz das fibroses pós-inflamatórias ${ }^{6}$ e definir as condições do caráter de automanutenção nas fibromatoses. De maneira semelhante, empreender uma pesquisa da expressão gênica das células conjuntivas nas fibroses pela utilização de técnicas de hibridização in situ ("mapping").

- Acompanhar com técnicas não invasivas (dosagens séricas) a evolução das fibroses de órgãos (fígado, pulmão, cartilagem, pele) no decurso de situações clínicas selecionadas. Trata-se de contribuir para estabelecer um diagnóstico e/ou prognóstico evolutivo útil na estratégia terapêutica das fibroses no homem ("monitoring").
Actuellement, la seule méthode facile à utiliser, et aussi la plus répandue, c'est le dosage radio-immunologique du P-III-P (propeptide amino-terminal du procollagène de type III) ${ }^{2}$. En effet, les dosages d'activé enzymatique sont encore techniquement délicats et souvent entachés d'erreurs. En outre, l'activité enzymatique circulante est rapidement détruite ou inhibée. Des résultats intéressants ont par contre été rapportés avec le dosage du P-III-P: le chevauchement des valeurs individuelles obtenues dans differentes pathologies hépatiques interdit l'utilisation de ce test à des fins diagnostiques. Cependant, associé à d'autres marqueurs, il peut être une aide au diagnostic en permettant par exemple de différencier la simple stéatose de l'hepátite alcoolique aiguë all cours de l'intoxication alcoolique, ou bien en cas d'hépatite virale chronique de suivre l'évolution vers l'hépatite chronique active $\mathrm{s}^{14}$. En effet, en général, le taux sérique de P-III-P semble être plus un marqueur d'activité de la fibrose qu'une marqueur de la quantité de fibrose. Cela explique les discordances rencontrées dans les études portant sur la cirrhose hépatique: en effet, le taux sera élevé en cas d'apposition récente de collagène de type III et donc de fibrose active, mais il ne sera pas élevé en cas de fibrose installée où prédomine alors le collagène de type I. D'autres peptides circulants sont en cours d'expertise et pourraient complèter sous peu la gamme des tests disponibles: procollagène I, fragments de collagène de type IV (7S, NC1).

En pratique, jusqu'à présent, aucune étude clinique n'a porté sur des groupes de patients homogènes, suivis dans le temps et expertisés simultanément avec tous les marqueurs de fibrose disponible, tissulaires et sériques. Il faut dévelcpper ce type d'étude et d'ores et déjà on peut affirmer que le clinicien ne peut plus avoir une attitude résignée devant la fribrose hépatique. La fibrose hépatique est une maladie autonome, qu'il doit dépister, évaluer et qu'il pourra dans un proche avenir traiter: en effet, des résultats intéressants ont été rapportés au moins chez l'animal avec de nouvelles molécules potentiellement antifibrosantes.

Pour conclure, il apparait clair que les recherches convergent maintenant vers trois objectifs:

- Établir les mécanismes cellulaires et moléculaires qui régulent, in vivo, la nature du dépôt matriciel dans les fibroses post-inflammatoires ${ }^{6}$ et définir les conditions de son caractère auto-entretenu dans les fibromatoses. De façon parallèle, une recherche de l'expression génique des cellules conjonctives est entreprise dans les fibroses par l'utilisation des techriques d'hybridation in situ ("mapping").

- Surveiller par des techniques non invasives (dosages sériques) l'évolution des fibroses d'organes (foie, poumon, cartilage, peau) au cours de situations 
Editorial. Grimaud JA, Druguet M, Hartmann D. Quand la fibrose hépatique devient une maladie... Revista da Sociedade Brasileira de Medicina Tropical 20: 189-192, Out-Dez, 1987

- Explorar as capacidades de degradação e de reversibilidade das fibroses pela análise de remodelação do tecido conjuntivo in vivo e in vitro. Trata-se de um dado curiosamente pouco documentado que as pesquisas, realizadas recentemente por diferentes laboratórios, devem trazer esclarecimentos a) pela identificação e utilização de "marcadores de degradação": soros imunes especificos de peptideos presentes no decurso da fibrólise ${ }^{4}$; b) pelo controle da transformação e da estabilidade, in vivo e in vitro, de complexos de matrizes acelulares isoladas do figado, facilmente acessiveis, suportes e autores da remodelação ${ }^{13}$ ("moving and removing").

Estes objetivos devem permitir a contribuição de maneira significativa ao rápido progresso no conhecimento das fibroses celulares, de sua capacidade de reversibilidade, de sua remodelação espontânea ou secundária à ação de moléculas com finalidade terapêutica.

\section{BIBLIOGRAPHIE}

1. Clément B, Grimaud JA, Campion JP, Deugnier Y, Guillouzo A. Cell types involved in collagen and fibronectin production in normal and fibrotic human liver. Hepatology 6: 225-234, 1986.

2. Cordier JF, Grimaud JA, Peyrol S, Mornex JF, Brune J. Aspects généraux des fibroses pulmonaires. Symposium GERP, 28-30 avril 1986, Publications INSERM (sous presse).

3. Demarchez M, Hartmann DJ, Prunieras M. An immunohistological study of the revascularization process in human skin transplanted onto the nude mouse. Transplantation 43: 896, 1987.

4. Emonard H, Peyrol S, Guerret-Stocker S, Druguet M, Griumaud JA. Antibody to CB-peptide, $\alpha_{2}$ CB $(3,5)$, from type I collagen is a tissular marker of matrix degradation in hepatic murine schistosomiasis. Cellular and Molecular Biology 32:633-640, 1986.

5. Frei A, Zimmermann A, Weigand $K$. The $\mathrm{N}$-terminal propeptide of collagen type III in serum reflects activity and degree of fibrosis in patients with chronic liver disease. Hepatology 4: 830-834, 1984.

6. Grimaud JA, Boros DL, Takiya C, Mathew RC, Emonard $\mathrm{H}$. Collagen isotypes, laminin and fibronectin in granulomas of the liver and intestine of $S$. mansoni infected mice. American Journal of Tropical Medicine and Hygiene 37: 332-345, 1987.

7. Grimaud JA, Druguet M, Peyrol S, Chevalier O, Herbage $\mathrm{D}$, El Badrawy $\mathrm{N}$. Collagen immunotyping in cliniques choisies. Il s'agit de contribuer à établir un diagnostic et/ou un pronostic évolutif utile dans la stratégie therapeutique des fibroses chez l'Homme ("monitoring").

- Explorer les capacités de dégradation et de réversibilité des fibroses par l'analyse du remodelage du tissu conjonctif in vivo et in vitro. Il s'agit d'une donnée curieusement peu documentée que les actions de recherche menées récemment par différents laboratoires doivent faire progresser significativement a) par l'identification et l'utilisation de "marqueurs de dégradation": immunsérums spécifiques de peptides présents au cours de la fibrolyse ${ }^{4}$; b) par le contrôle du devenir et de la stabilite, in vivo et in vitro, de complexes matriciels accellulaires isolés du foie, facilement acessible, supports et acteurs du remodelage 13 ("moving and removing").

Ces objectifs doivent permettre de contribuer de façon significative à un progrès rapide dans la connaissance des fibroses tissulaires, de leur capacité de réversibilité, de leur remodelage spontané ou secondaire à l'action de molécules à visée thérapeutique.

human liver.Light and electron microscope study.Journal of Histochemistry and Cytochemistry 28:1 145-1 156,1980.

8. Grimaud JA, Druguet M, Peyrol S, Guerret S. Collagen. In: "Methods of Enzymatic Analysis", vol. IX Proteins and Peptides, HU. Bergmeyer (ed.) VCH Publisher, Weinheim (Federal Republic of Germany), pp. 186-201, 1986.

9. Guerret S, Rojkind M, Druguet M, Chevallier M, Grimaud JA. Immunohistochemical micromethods for the measurement of specific collagen types in human liver biopsies. (soumis pour publication).

10. Hahn EG. Blood analysis for liver fibrosis. Joumal of Hepatology 1: 67-73, 1984.

11. Hartmann DJ, Trinchet JC, Galet B, Callard P, Nusgens B, Ville G, Lapière CM, Beaugrand M. Radioimmunoassays of human type I and III collagens in serum of patients with alcoholic and non alcoholic liver diseases. Clinical Chemistry 32: 1143, 1986.

12. Lopez de Leon A, Rojkind M. A simple micromethod for collagen and total protein determination in formalin-fixed paraffin embedded sections. Journal of Histochemistry and Cytochemistry 33: 737-743, 1985.

13. Takiya C, Lenoir J, Grimaud JA. Isolation and preparation of acellular schistosomal granuloma (periovular matricial complex PMC).Cellular and Molecular Biology 32: 647-653, 1986.

14. Trinchet JC, Hartmann DJ, Pateron D, Galet B, Ville G, Callard P, Beaugrand M. Les dosages du CI et du PIIIP sériques remplaceront-ils la biopsie du foie dans le suivi des malades atteints d'hépatite chronique? Gastroenterologie Clinique et Biologique 10: 19 A, 1986. 